\title{
Comparison of Three-Dimensional Speckle Tracking Echocardiography and Two-Dimensional Echocardiography for Evaluation of Left Atrial Size and Function in Healthy Volunteers (Results from the MAGYAR-Healthy Study)
}

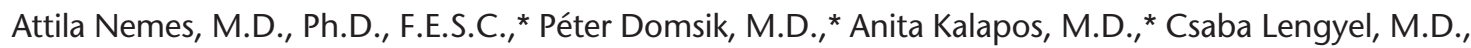 \\ Ph.D. $\dagger$ Andrea Orosz, M.D., $\ddagger$ and Tamás Forster, M.D., Ph.D., D.Sc., F.E.S.C., F.A.C.C.* \\ *2nd Department of Medicine and Cardiology Center, Medical Faculty, Albert Szent-Györgyi Clinical Center, \\ University of Szeged, Szeged, Hungary; $\uparrow 1$ st Department of Medicine, Medical Faculty, Albert Szent-Györgyi \\ Clinical Center, University of Szeged, Szeged, Hungary; and $\ddagger$ Department of Pharmacology and \\ Pharmacotherapy, Medical Faculty, University of Szeged, Szeged, Hungary
}

Objective: Noninvasive accurate assessment of left atrial (LA) size and function is an essential requirement in daily clinical practice. Real time three-dimensional (3D) echocardiography (RT3DE) with direct volumetric method has been found to be a highly accurate and reproducible noninvasive tool for the evaluation of LA dimensions and functional properties. Three-dimensional speckle tracking echocardiography (3DSTE) has just been introduced for volumetric assessments, which uses different, as called "block-matching" algorithm by strain analysis. This study was designed to compare two-dimensional (2D) echocardiography with 3DSTE for calculation of LA volumes and assessment of LA functional properties in healthy subjects. Methods: This study comprised of randomly selected 35 healthy subjects (40.9 \pm 10.9 years, 20 men) in sinus rhythm, they all had undergone standard transthoracic 2D echocardiographic Doppler study extended with 3DSTE. Results: Two-dimensional echocardiography- and 3DSTE-derived minimum $\left(V_{\min }\right)$ and maximum $\left(V_{\max }\right)$ LA volumes and LA volume before atrial contraction $\left(V_{\text {pre }} \mathrm{A}\right)$ did not differ significantly. Calculated functional LA properties were also compared. Good correlations were found between both techniques for $V_{\max }(r=0.93, P<0.0001), V_{\min }$ $(r=0.62, P<0.0001)$, and $V_{\text {pre } A}(r=0.74, P<0.0001)$. Conclusions: It may be stated that 3DSTE seems to be feasible in detection of cyclic changes in LA volumes and calculation of its functional properties is comparable to 2D echocardiography. (Echocardiography 2014;31:865-871)

Key words: left atrium, function, two-dimensional, three-dimensional, echocardiography, speckle tracking

Noninvasive accurate assessment of left atrial (LA) size and function is an essential requirement in daily clinical practice. ${ }^{1}$ Real time three-dimensional (3D) echocardiography (RT3DE) with direct volumetric method has been found to be a highly accurate and reproducible noninvasive tool for the evaluation of LA dimensions and functional properties. ${ }^{2,3}$ 3D speckle tracking echocardiography (3DSTE) has just been introduced for volumetric assessments, which uses different, as called "block-matching" algorithm by

Address for correspondence and reprint requests: Attila Nemes, M.D., Ph.D., F.E.S.C., 2nd Department of Medicine and Cardiology Center, Medical Faculty, Albert Szent-Györgyi Clinical Center, University of Szeged, H-6720 Szeged, Korányi fasor 6, Hungary, P.O. Box 427. Fax: 36-62-544568;

E-mail: nemes.attila@med.u-szeged.hu strain analysis. ${ }^{4,5}$ In a recent study, RT3DE and 3DSTE methods for quantification of LA volumes and function were found to be comparable, reproducible and interchangeable. ${ }^{6}$

It is known that two-dimensional (2D) echocardiography is one of the most widely used standardized imaging tool for the evaluation of LA dimensions. ${ }^{7}$ Therefore, this study was designed to compare 2D echocardiography with 3DSTE for calculation of LA volumes and assessment of LA functional properties in healthy subjects.

Patients and Methods:

Patient Population:

Recently, a new study named MAGYARHealthy Study (Motion Analysis of the heart and $\mathbf{G r e a t}$ vessels bY three-dimension $\mathbf{A l}$ speckle 
tRacking echocardiography in Healthy subjects) has been organized at the Cardiology Center of University of Szeged, Hungary to evaluate usefulness, diagnostic and prognostic value of 3DSTE-derived volumetric, strain, rotation, etc. parameters in healthy volunteers ("magyar" means "hungarian" in hungarian language). The present work comprised randomly selected 35 healthy subjects (mean age: $40.9 \pm 10.9$ years, 20 men) in sinus rhythm, they all had undergone standard transthoracic Doppler 2D echocardiographic study extended with 3DSTE. None of the subjects had known disease or any factor affecting results. Informed consent was obtained from each patient and the study protocol conformed to the ethical guidelines of the 1975 Declaration of Helsinki, as reflected in a priori approval by the institution's human research committee.

\section{Two-Dimensional Echocardiography:}

Complete routine 2D echocardiography was undertaken with the patient lying in the left lateral decubitus position with quiet respiration using both apical and parasternal views. ${ }^{3}$ Doppler 2D echocardiographic studies were performed with a Toshiba Artida echocardiography equipment using a PST-30SBP (1-5 MHz) phased-array transducer (Toshiba Medical Systems, Tokyo, Japan). For evaluation of LA size and function, the following parameters were measured on 2D images (Fig. 1):

(1) Left atrial area at apical four-chamber view $(4 \mathrm{CH})(\mathrm{A} 1)$ by manual tracing of LA endocardial border. The superior border of atrial outline was a straight line connecting both sides of the mitral leaflet base attachment points. Both LA appendage and pulmonary veins were excluded when visualized, and

(2) Left atrial area at apical two-chamber view $(2 \mathrm{CH})(\mathrm{A} 2)$ with same tracing, and

(3) LA long axis (L) defined as the distance of the perpendicular line measured from the middle of the plane of mitral annulus to the superior aspect of LA in both apical $4 \mathrm{CH}$ and $2 \mathrm{CH}$ views and the shortest of both lines was used.

\section{Three-Dimensional Speckle Tracking Echocardiography:}

Three-dimensional speckle tracking echocardiography has been performed using a $1-4 \mathrm{MHz}$ matrix phased-array transducer (PST-25SX) with the same ultrasound system. ${ }^{6}$ Within a single breath hold and during a constant RR interval, 6 wedge-shaped subvolumes were acquired from an apical window to create full volume 3D datasets including LA. For optimal LA border delineation, depth was increased and sector width was decreased. Chamber quantification by 3DSTE was performed using 3D Wall Motion Tracking software version 2.7 (Toshiba Medical Systems). $3 \mathrm{D}$ echocardiographic datasets were displayed in apical $2 \mathrm{CH}$ and $4 \mathrm{CH}$ views and 3 short-axis views at different LA levels from the base to the apex. The endocardial border was traced by setting multiple reference points by the user starting at the base of LA at the mitral valve level on the septal side, where anterior mitral leaflet origins going toward the apex on counterclockwise direction till the origin of the mitral posterior leaflet excluding the LA appendage and the pulmonary veins from the LA cavity. Measurements were performed firstly on apical $4 \mathrm{CH}$ view,
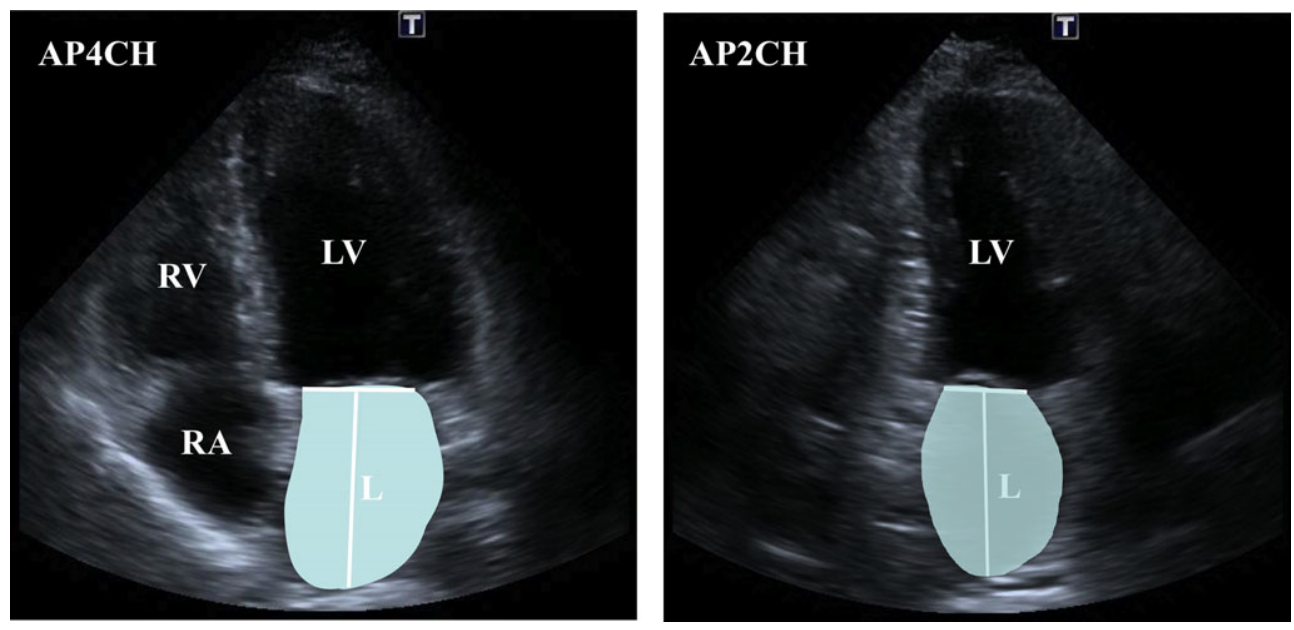

Figure 1. Calculation of left atrial (LA) volume using $2 \mathrm{D}$ echocardiography by manual tracing of LA endocardial border at apical four-chamber (AP4CH) and two-chamber views (AP2CH). L is the long axis, then apply the formula. $L V=$ left ventricle; $R A=$ right atrium; RV = right ventricle. 
later on $2 \mathrm{CH}$ view. After detection of the myocardial borders at the end-diastolic reference frame, the user could correct the LA shape, if necessary. The 3D wall-motion tracking was then automatically performed through the entire cardiac cycle. $3 \mathrm{D}$ cast and volumetric data of LA at different moments of cardiac cycle were generated by the software (Fig. 2).

\section{Evaluation of LA Volumes:}

Left atrial volumes were calculated at the same phases by 2D echocardiography and 3DSTE according to the formula : Volume $=8(\mathrm{~A} 1)$ $(\mathrm{A} 2) / 3 \pi(\mathrm{L})$ at 3 phases of ventricular cardiac cycle:

(1) maximum volume $\left(V_{\max }\right)$ at end-systole, the time at which atrial volume was largest just before mitral valve opening,

(2) minimum volume $\left(V_{\min }\right)$ : at end-diastole, the time at which atrial volume at its nadir before mitral valve closure

(3) volume before atrial contraction $\left(V_{\text {pre }} \mathrm{A}\right)$ : the last frame before mitral valve reopening or at time of P-wave on ECG.

From the three volumes, the following indices of LA function were calculated by both methods ${ }^{8,9}$.
(1) Total atrial stroke volume (TASV): $\mathrm{V}_{\max }-\mathrm{V}_{\min }$.

(2) Total atrial emptying fraction (TAEF): TASV $/ \mathrm{V}_{\max } \times 100$.

(3) Active atrial stroke volume (AASV): $V_{\text {pre }} \mathrm{A}$ $-V_{\text {min }}$.

(4) Active atrial emptying fraction (AAEF): AASV/V $V_{\text {pre A }} \times 100$

(5) Passive atrial stroke volume (PASV): $V_{\text {max }}-V_{\text {pre } A}$

(6) Passive atrial emptying fraction (PAEF): $\left(V_{\max }-V_{\text {pre } A}\right) / V_{\max } \times 100$

\section{Statistical Analysis:}

Data are reported as mean \pm standard deviation. A value of $P<0.05$ was considered to be statistically significant. MedCalc software was used for statistical calculations (MedCalc, Mariakerke, Belgium). Paired sample $t$-test was performed to determine whether the difference in the values between 2 methods was significant or not. Pearson's coefficient was used for inter-observer and intra-observer correlations. Intra- and interobserver agreements were studied according to Bland and Altman method. ${ }^{10}$ In a recent study, mean difference for $V_{\text {max }}, V_{\min }$, and $V_{\text {pre }}$ A used the same 2D method was $6.03 \pm 9.43 \mathrm{~mL}$, $-0.26 \pm 3.17 \mathrm{~mL}$, and $1.71 \pm 4.31 \mathrm{~mL}$ with

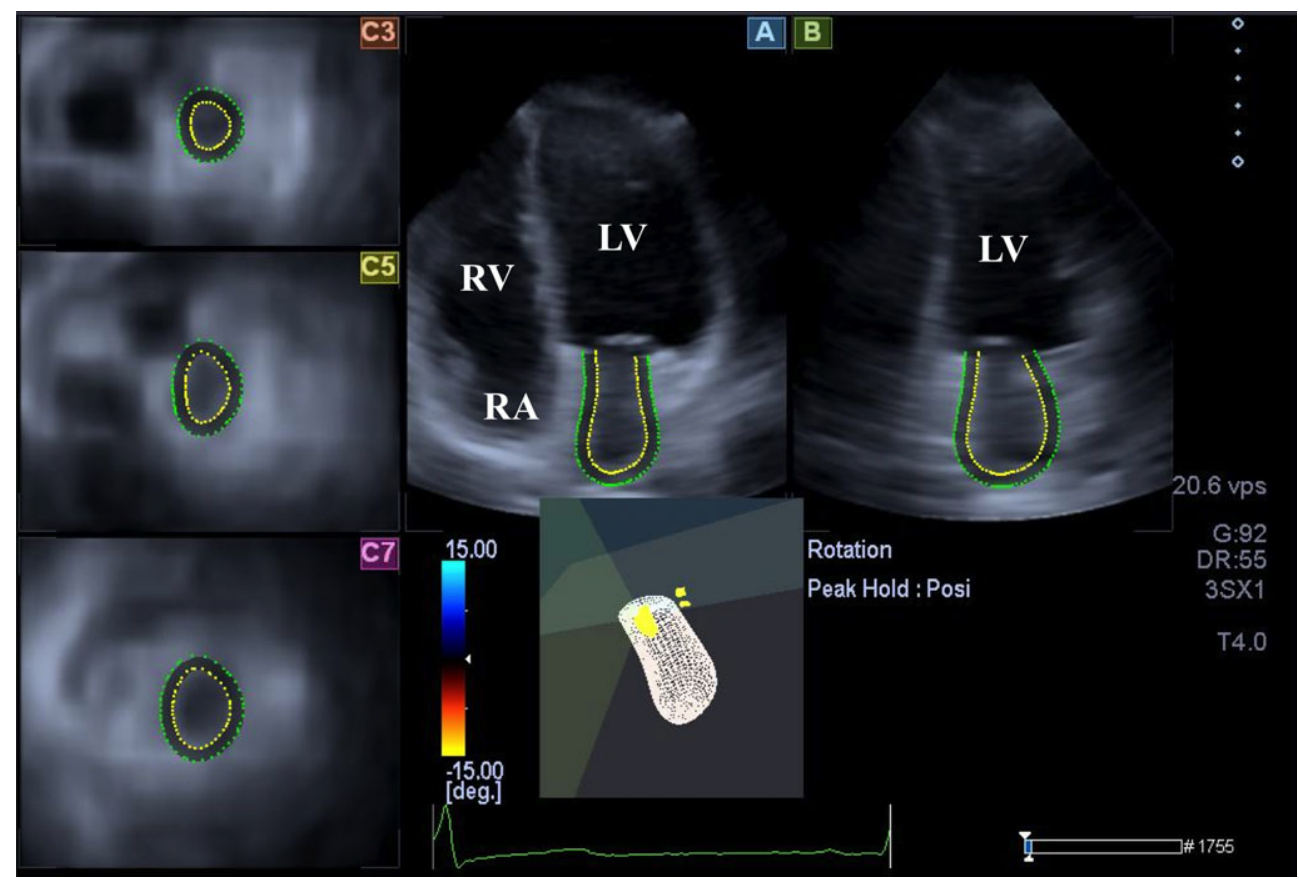

Figure 2. Three-dimensional (3D) echocardiographic dataset is displayed in apical four-chamber. A. and two-chamber views B. and 3 short-axis views in the basal C3., mid-atrial C5., and apical C7. regions, respectively. 3D LA cast is also presented. LV $=$ left ventricle; $\mathrm{RA}=$ right atrium; $\mathrm{RV}=$ right ventricle. 
correlation coefficients $0.93,0.98$, and 0.97 $(P<0.0001)$, respectively. ${ }^{3}$

Results:

\section{Volumetric 3DSTE and 2D Echocardiographic} Data:

Volumetric data and functional parameters of LA are presented in Table I.

\section{Correlations between 3DSTE-Derived and 2D Echocardiographic Data:}

A good correlation was found between both techniques for $V_{\max }(r=0.93, P<0.0001), V_{\min }$ $(r=0.62, P<0.0001)$, and $V_{\text {pre }} A(r=0.74$, $P<0.0001)$. Similar correlations were found between TASV $(r=0.85, \quad P<0.0001)$, TAEF $(r=0.81, \quad P<0.0001), \quad$ PASV $(r=0.67, \quad P<$ $0.0001)$, PAEF $(r=0.74, \quad P<0.0001), \quad$ AASV $(r=0.71, \quad P<0.0001)$, and AAEF $(r=0.69$, $\mathrm{P}<0.0001)$.

\section{Reproducibility of Measurements:}

The mean \pm standard deviation difference in values obtained by 2 measurements of the same observer for the measurements of 3DSTE-derived $\mathrm{V}_{\max ,} \mathrm{V}_{\min }$ and $\mathrm{V}_{\text {pre }} \mathrm{A}$ was $-0.8 \pm 9.3 \mathrm{~mL}$, $-0.5 \pm 6.6 \mathrm{~mL}$, and $-1.5 \pm 8.0 \mathrm{~mL}$, respectively. Correlation coefficients between measurements of the same observer were $0.76,0.79$, and

\section{TABLE I}

Left Atrial Dimensions and Functional Parameters Using TwoDimensional Echocardiography and Three-Dimensional Speckle Tracking Echocardiography in Healthy Controls

\begin{tabular}{|c|c|c|c|}
\hline & 2D Echocardiography & 3DSTE & $\mathrm{P}$ \\
\hline \multicolumn{4}{|c|}{ Calculated LA volumes } \\
\hline $\mathrm{V}_{\max }(\mathrm{mL})$ & $35.5 \pm 0.7$ & $36.6 \pm 6.5$ & NS \\
\hline$V_{\min }(m L)$ & $14.9 \pm 0.4$ & $16.5 \pm 5.0$ & NS \\
\hline$V_{\text {pre A }}(\mathrm{mL})$ & $23.8 \pm 4.8$ & $24.0 \pm 6.1$ & NS \\
\hline \multicolumn{4}{|c|}{ LA stroke volumes } \\
\hline TASV (mL) & $20.6 \pm 5.9$ & $20.1 \pm 5.0$ & NS \\
\hline PASV $(\mathrm{mL})$ & $12.1 \pm 4.3$ & $12.3 \pm 5.0$ & NS \\
\hline AASV (mL) & $8.8 \pm 3.8$ & $7.5 \pm 2.9$ & NS \\
\hline \multicolumn{4}{|c|}{ LA emptying fractions } \\
\hline TAEF (\%) & $57.6 \pm 10.0$ & $55.2 \pm 10.7$ & NS \\
\hline PAEF (\%) & $32.0 \pm 11.9$ & $34.6 \pm 11.3$ & NS \\
\hline AAEF (\%) & $37.0 \pm 13.8$ & $31.5 \pm 9.5$ & $<0.05$ \\
\hline
\end{tabular}

$\mathrm{LA}=$ left atrial; $\mathrm{AAEF}=$ active atrial emptying fraction; $\mathrm{AASV}=$ active atrial stroke volume; PAEF = passive atrial emptying fraction; PASV = passive atrial stroke volume; TAEF = total atrial emptying fraction; TASV = total atrial stroke volume; $\mathrm{V}_{\max }=$ maximum volume at end-systole; $\mathrm{V}_{\min }=$ minimum volume at end-diastole; $\mathrm{V}_{\text {pre } \mathrm{A}}=$ volume before atrial contraction; $2 \mathrm{D}=$ two-dimensional; $3 \mathrm{DSTE}=$ three-dimensional speckle tracking echocardiography.
$0.82(\mathrm{P}<0.0001)$, respectively (Figs. $3-5)$ (intraobserver agreement). The mean \pm standard deviation difference in values obtained by two observers for 3DSTE-derived $\mathrm{V}_{\max }, \mathrm{V}_{\min }$, and $\mathrm{V}$ pre $A$ was $0.9 \pm 9.3 \mathrm{~mL},-0.3 \pm 7.4 \mathrm{~mL}$, and $-0.5 \pm 7.3 \mathrm{~mL}$, respectively. Correlation coefficient between these independent measurements of 2 observers were $0.75,0.77$, and 0.82 $(\mathrm{P}<0.0001)$, respectively (Figs. $3-5)$ (interobserver agreement).

\section{Discussion:}

Results of this study could suggest feasibility of 3DSTE in detection of cyclic changes in LA volumes and calculation of its functional properties. To the best of authors' knowledge this is the first study to directly compare 3DSTE with 2D echocardiography for the evaluation of volumetric and functional LA parameters.

Left atrial volume has been identified as a potential biomarker for different cardiovascular diseases. ${ }^{11}$ However, during evaluation of volumes phasic function of the LA should be respected including:

(1) a reservoir during LV systole (Reservoir function)

(2) a conduit for blood transiting from the pulmonary veins to the LV during early diastole (Conduit function)

(3) an active contractile chamber that augments LV ventricular filling in late diastole (Active contraction).

These phases of the LA function could be characterized by several parameters based on accurate measurement of maximum and minimum $L A$ volumes $\left(V_{\max }\right.$ and $\left.V_{\text {min }}\right)$ and $L A$ volume before atrial contraction $\left.\left(V_{\text {pre } A}\right)\right)^{2,3} 2 \mathrm{D}$ echocardiographic evaluation of LA is standardized for a decade and provides reasonable assessment of LA volumes, ${ }^{7}$ although all measurement methods underestimate LA volumes with $14-37 \%$ as compared to computed tomography $(\mathrm{C} T)^{12}$ and magnetic resonance imaging (MRI). ${ }^{13} \mathrm{~A}$ minor nonsignificant improvement in the evaluation of LA could be obtained by the early generation multiplane 3D echocardiographic method based on 90 images acquired by apical probe rotation. ${ }^{12}$ Anwar et al. ${ }^{2,3}$ demonstrated the feasibility and reproducibility of direct volumetric RT3DE in the detection of cyclic changes in LA volumes and calculation of its function as compared to 2D echocardiography.

Recently, 3DSTE has been introduced, firstly to provide a comprehensive assessment of LV function through strain analysis in four dimensions. ${ }^{6,14-16}$ 3DSTE uses different algorithm as 

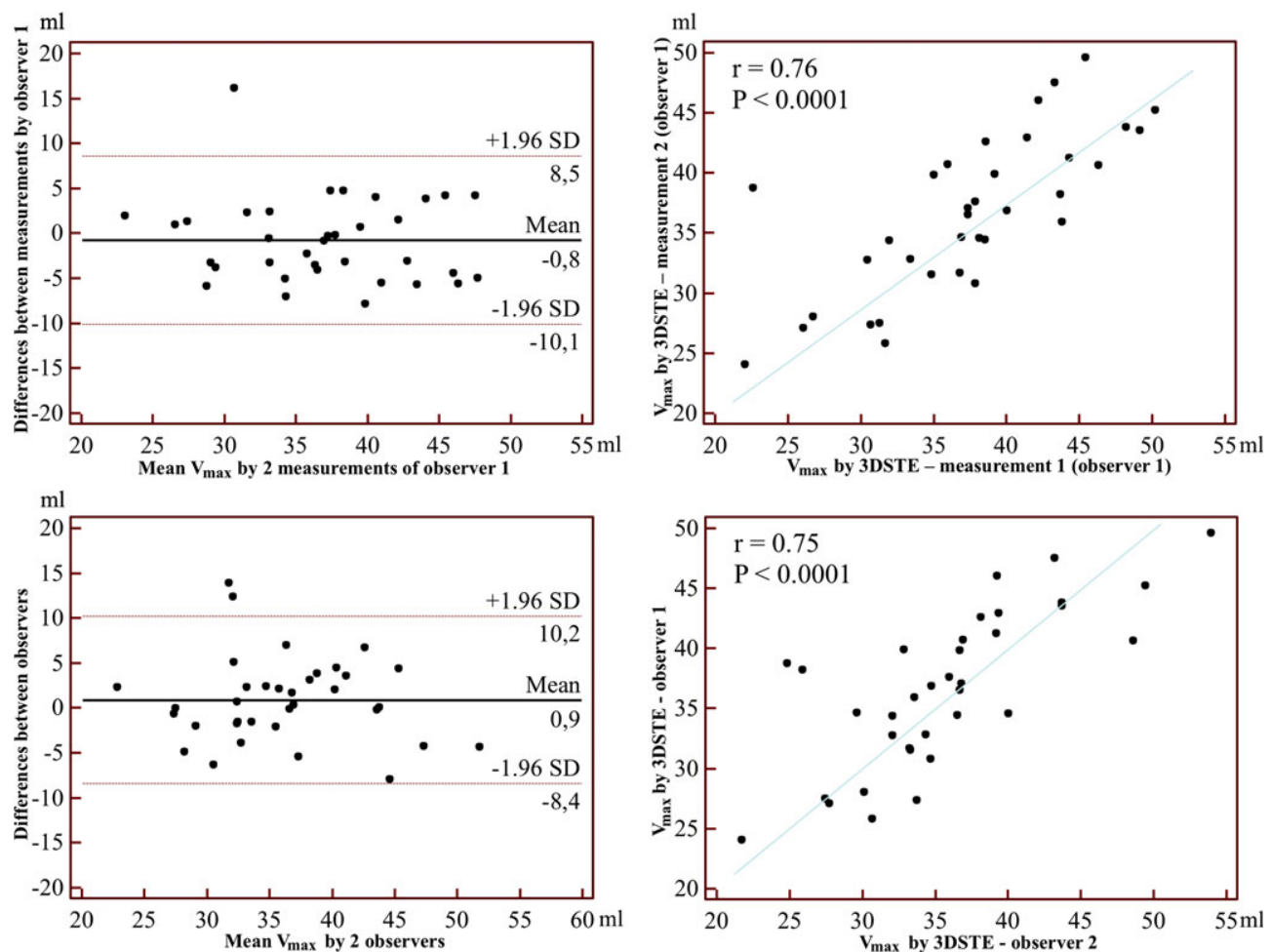

Figure 3. Intra-observer (upper graphs) and inter-observer (lower graphs) agreements and correlations for measuring $V_{\text {max }}$ by three-dimensional speckle tracking echocardiography (3DSTE) are presented. $\mathrm{V}_{\max }=$ maximum left atrial volume.
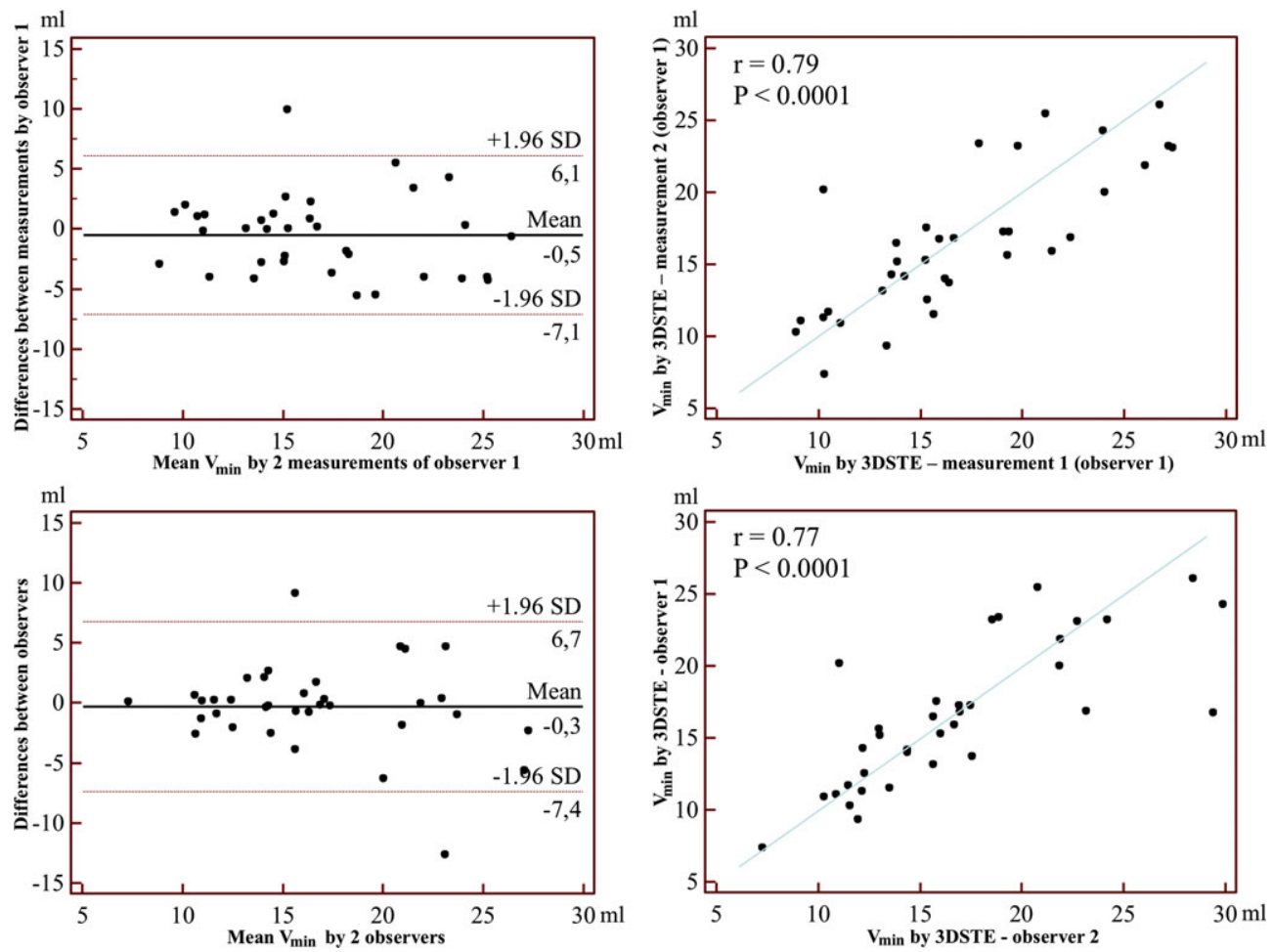

Figure 4. Intra-observer (upper graphs) and inter-observer (lower graphs) agreements and correlations for measuring $V_{\text {min }}$ by three-dimensional speckle tracking echocardiography (3DSTE) are presented. $\mathrm{V}_{\min }=$ minimum left atrial volume. 

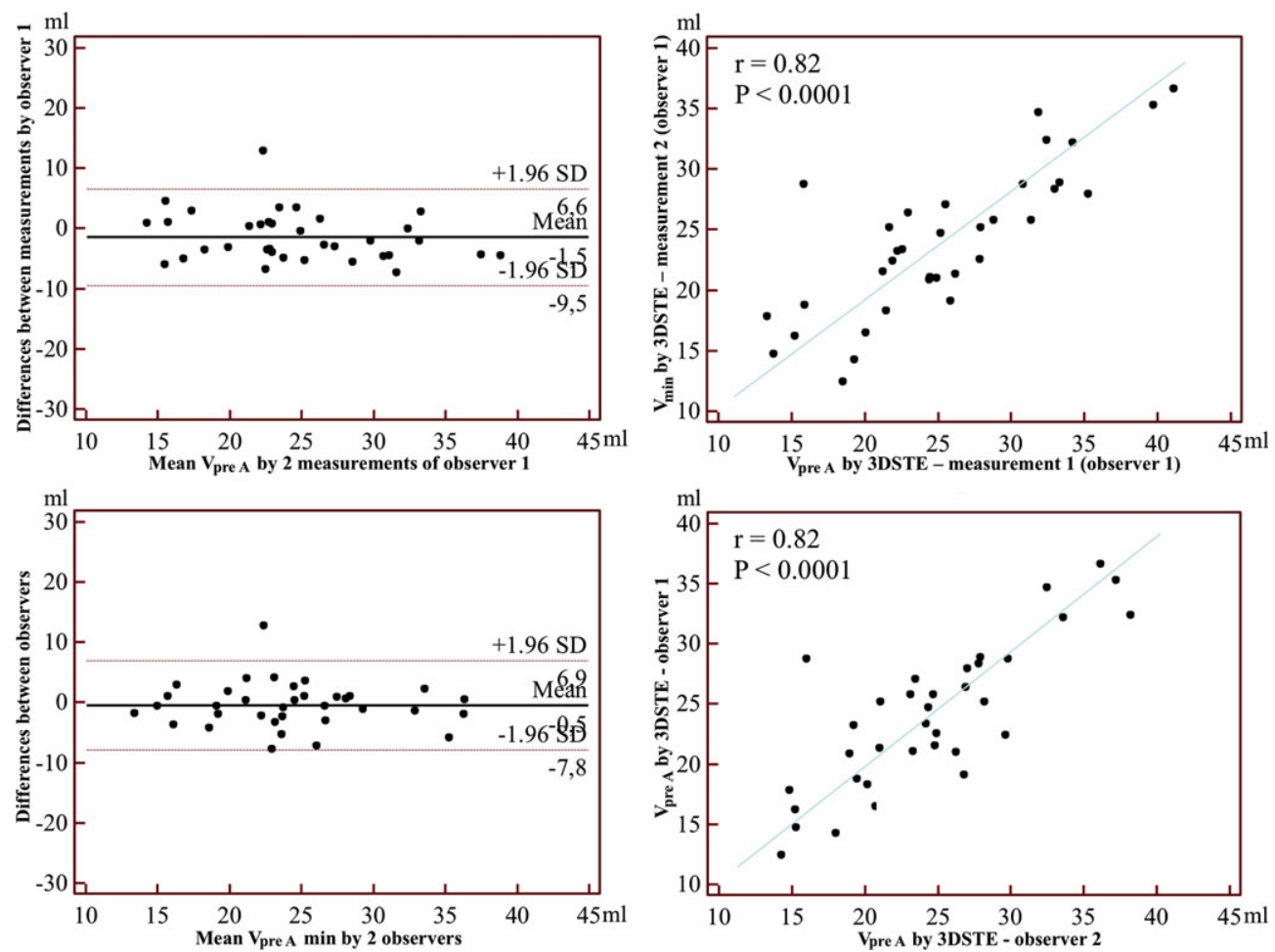

Figure 5. Intra-observer (upper graphs) and inter-observer (lower graphs) agreements and correlations for measuring $V_{\text {pre }} \mathrm{A}$ by three-dimensional speckle tracking echocardiography (3DSTE) are presented. $\mathrm{V}_{\text {pre } \mathrm{A}}=$ volume before atrial contraction.

compared to RT3DE which is based on block matching of the myocardial speckles of the endocardial border during their motion from frame to frame. ${ }^{17}$ This new 3DSTE technique for LV volume measurements has been validated and its superior accuracy and reproducibility over previously used 2DSTE have even been demonstrated by MRI. ${ }^{14}$ Good intra-observer, interobserver, and test-retest reliability were found supporting the use of 3DSTE for routine evaluation of LV volumes and ejection fraction. ${ }^{16}$ Kleijn et al. $^{6}$ were the first to demonstrate that $3 \mathrm{D}$ echocardiography with direct volumetric (RT3DE) and speckle tracking methods (3DSTE) give comparable and reproducible quantification of LV and LA volumes and function, making interchangeable application a viable option in daily clinical practice. Nagaya et al. ${ }^{18}$ aimed to validate the accuracy of 3DSTE and 2DSTE for the assessment of LA volume and function by comparison with 3D-CT. 3DSTE allowed more accurate measurement of LA volume and function than 2DSTE and had high reproducibility. ${ }^{18}$ Results of this study are in agreement with these findings demonstrating feasibility and reproducibility of 3DSTE in the evaluation of LA volumes and functional properties. Good correlations were found between measured volumetric and calculated functional properties between 2D echocardiography and 3DSTE. Despite image quality for 3DSTE is worse due to low temporal and spatial image resolutions than 2D echocardiography, but 3D echo visualizes LA as it truly is: 3D organ. This advantage of 3DSTE (and RT3DE) could highlight our attention on this new technology which probably will be a milestone in the echocardiographic evaluation of LA volumes and functional properties.

\section{Limitation Section:}

However, some important limitations should be taken into consideration analyzing our results:

(1) Left atrial appendage for calculation of LA volume and function was not included due to its variability in shape and lack of standard figures (3).

(2) As mentioned previously, current image quality obtained by 3DSTE is worse than for 2D echocardiography due to low temporal and spatial image resolutions.

(3) This was a single-center experience and limited by a relatively small number of healthy volunteers. The study would have been statistically stronger, if larger number of subjects had been evaluated. Caution should be taken when interpreting the results observed. 
(4) Although 3DSTE seems to be feasible for the assessment of strain and rotational characteristics of LA, this study did not aimed to evaluate and validate these parameters. $^{19}$

\section{Conclusions:}

Three-dimensional speckle tracking echocardiography seems to be feasible in detection of cyclic changes in LA volumes and calculation of its functional properties is comparable to $2 \mathrm{D}$ echocardiography.

Acknowledgments: Dr. Attila Nemes holds a János Bolyai Research Fellowship (Budapest, Hungary).

\section{References}

1. European Heart Rhythm Association (EHRA), European Cardiac Arrhythmia Society (ECAS), American College of Cardiology (ACC), American Heart Association (AHA), Society of Thoracic Surgeons (STS), Calkins H, Brugada J, Packer DL, et al: HRS/EHRA/ECAS expert Consensus Statement on catheter and surgical ablation of atrial fibrillation: Recommendations for personnel, policy, procedures and follow-up. A report of the Heart Rhythm Society (HRS) Task Force on catheter and surgical ablation of atrial fibrillation. Heart Rhythm 2007; 4:816-861.

2. Anwar AM, Geleijnse ML, Soliman OI, et al: Left atrial Frank-Starling law assessed by real-time, three-dimensional echocardiographic left atrial volume changes. Heart 2007;93:1393-1397.

3. Anwar AM, Soliman OI, Geleijnse ML, et al: Assessment of left atrial volume and function by real-time threedimensional echocardiography. Int / Cardiol 2008; 123:155-161.

4. Ammar KA, Paterick TE, Khandheria BK, et al: Myocardial mechanics: Understanding and applying three-dimensional speckle tracking echocardiography in clinical practice. Echocardiography 2012;29:861-872.

5. Nemes A, Kalapos A, Domsik P, et al: Three-dimensional speckle-tracking echocardiography - a further step in non-invasive three-dimensional cardiac imaging. Orv $\mathrm{He}$ til 2012;153:1570-1577.

6. Kleijn SA, Aly MF, Terwee CB, et al: Comparison between direct volumetric and speckle tracking methodologies for left ventricular and left atrial chamber quantification by three-dimensional echocardiography. Am / Cardiol 2011;108:1038-1044.

7. Lang RM, Bierig M, Devereux RB, et al, Chamber Quantification Writing Group, American Society of Echocardiography's Guidelines and Standards Committee, European Association of Echocardiography: Recommendations for chamber quantification: A report from the American
Society of Echocardiography's Guidelines and Standards Committee and the Chamber Quantification Writing Group, developed in conjunction with the European Association of Echocardiography, a branch of the European Society of Cardiology. I Am Soc Echocardiogr 2005;18:1440-1463.

8. Poutanen $T$, Jokinen $E$, Sairanen $H$, et al: Left atrial and left ventricular function in healthy children and young adults assessed by three dimensional echocardiography. Heart 2003;89:544-549.

9. Blondheim DS, Osipov A, Meisel SR, et al: Relation of left atrial size to function as determined by transesophageal echocardiography. Am J Cardiol 2005;96:457-463.

10. Bland JM, Altman DG: Statistical methods for assessing agreement between two methods of clinical measurement. Lancet 1986;1:307-310.

11. Leung DY, Boyd A, Ng AA, et al: Echocardiographic evaluation of left atrial size and function: Current understanding, pathophysiologic correlates, and prognostic implications. Am Heart / 2008;156:1056-1064.

12. Al-Mohaissen MA, Kazmi MH, Chan KL, et al: Validation of two-dimensional methods for left atrial volume measurement: A comparison of echocardiography with cardiac computed tomography. Echocardiography 2013;30: 1135-1142.

13. Rodevan $\mathrm{O}$, Bjornerheim $\mathrm{R}$, Ljosland $\mathrm{M}$, et al: Left atrial volumes assessed by three- and two-dimensional echocardiography compared to MRI estimates. Int I Card Imaging 1999;15:397-410.

14. Nesser HJ, Mor-Avi V, Gorissen W, et al: Quantification of left ventricular volumes using three-dimensional echocardiographic speckle tracking: Comparison with MRI. Eur Heart J 2009;30:1565-1573.

15. Maffessanti F, Nesser HJ, Weinert L, et al: Quantitative evaluation of regional left ventricular function using three-dimensional speckle tracking echocardiography in patients with and without heart disease. Am J Cardiol 2009; 104:1755-1762.

16. Kleijn SA, Aly MF, Terwee CB, et al: Reliability of left ventricular volumes and function measurements using threedimensional speckle tracking echocardiography. Eur Heart J Cardiovasc Imaging 2012;13:159-168.

17. Takeguchi T, Nishiura M, Abe Y, et al: Practical considerations for a method of rapid cardiac function analysis based on three-dimensional speckle tracking in a threedimensional diagnostic ultrasound system. I Med Ultrasonics 2010;37:41-49.

18. Nagaya M, Kawasaki M, Tanaka R, et al: Quantitative validation of left atrial structure and function by twodimensional and three-dimensional speckle tracking echocardiography: A comparative study with threedimensional computed tomography. I Cardiol 2013; 62:188-194.

19. Chadaide S, Domsik P, Kalapos A, et al: Three-dimensional speckle tracking echocardiography-derived left atrial strain parameters are reduced in patients with atrial fibrillation (results from the MAGYAR-Path Study). Echocardiography 2013;30:1078-1083. 